\title{
Expression of ceramide-metabolising enzymes in subcutaneous and intra-abdominal human adipose tissue
}

\author{
Maria Kolak , Joanna Gertow ${ }^{1}$, Jukka Westerbacka ${ }^{2}$, Scott A Summers ${ }^{3}$, Jan Liska ${ }^{4}$, Anders Franco-Cereceda ${ }^{4}$, \\ Matej Orešič ${ }^{5}$, Hannele Yki-Järvinen ${ }^{2}$, Per Eriksson ${ }^{1}$ and Rachel M Fisher ${ }^{1 *}$
}

\begin{abstract}
Background: Inflammation and increased ceramide concentrations characterise adipose tissue of obese women with high liver fat content compared to equally obese women with normal liver fat content. The present study characterises enzymes involved in ceramide metabolism in subcutaneous and intra-abdominal adipose tissue.

Methods: Pathways leading to increased ceramide concentrations in inflamed versus non-inflamed adipose tissue were investigated by quantifying expression levels of key enzymes involved in ceramide metabolism.

Sphingomyelinases (sphingomyelin phosphodiesterases SMPD1-3) were investigated further using immunohistochemistry to establish their location within adipose tissue, and their mRNA expression levels were determined in subcutaneous and intra-abdominal adipose tissue from both non-obese and obese subject.

Results: Gene expression levels of sphingomyelinases, enzymes that hydrolyse sphingomyelin to ceramide, rather than enzymes involved in de novo ceramide synthesis, were higher in inflamed compared to non-inflamed adipose tissue of obese women (with high and normal liver fat contents respectively). Sphingomyelinases were localised to both macrophages and adipocytes, but also to blood vessels and to extracellular regions surrounding vessels within adipose tissue. Expression levels of SMPD3 mRNA correlated significantly with concentrations of different ceramides and sphingomyelins. In both non-obese and obese subjects SMPD3 mRNA levels were higher in the more inflamed intra-abdominal compared to the subcutaneous adipose tissue depot.
\end{abstract}

Conclusions: Generation of ceramides within adipose tissue as a result of sphingomyelinase action may contribute to inflammation in human adipose tissue.

Keywords: Adipose tissue, Ceramide, Human, Inflammation, Sphingomyelinase

\section{Introduction}

Low grade systemic inflammation and insulin resistance often occur together, and adipose tissue is a site of inflammation in the insulin resistant state. In adipose tissue of obese and insulin resistant subjects macrophage number and inflammatory cytokine production are increased, while adiponectin production is decreased [1]. The mechanisms causing macrophage recruitment into adipose tissue are unclear, although adipocyte size has been implicated [1]. Furthermore, macrophages are often found clustered in

\footnotetext{
* Correspondence: rachel.fisher@ki.se

${ }^{1}$ Atherosclerosis Research Unit, Department of Medicine (Solna), Center for Molecular Medicine, Karolinska Institutet, Stockholm, Sweden

Full list of author information is available at the end of the article
}

"crown-like structures" surrounding individual adipocytes [2,3], which appear to be dead [2], suggesting that dead/ dying adipocytes may trigger macrophage influx, but the reason for adipocyte death is unknown. Another theory is that hypoxia may develop in regions of obese adipose tissue distant from the vasculature, causing hypoxic adipocytes to produce inflammatory cytokines and/or to die thus triggering macrophage infiltration [4]. An interesting possibility is that there are similarities between inflammatory pathways in obese/insulin resistant adipose tissue and the atherosclerotic artery wall. For example, oxidized lipid epitopes within adipose tissue might attract macrophages, or particular lipids (or their metabolites) might interfere with signalling, but this remains to be established.

\section{Biomed Central}


The degree of insulin resistance, rather than the degree of obesity, is of critical importance for the inflammatory status of adipose tissue [5]. Since fat accumulation in the liver predicts insulin resistance better than obesity [6], we postulated previously that hepatic fat content might distinguish between obese subjects who develop insulin resistance and adipose tissue inflammation and those who do not. This hypothesis was supported by investigation of adipose tissue from obese women with differing liver fat content [3]. We found greater expression of macrophagerelated genes and lower expression of adiponectin in subcutaneous adipose tissue from obese women with a high liver fat content compared to obese women with a normal liver fat content, but with comparable degrees of obesity. Furthermore, concentrations of certain ceramides and sphingomyelins were higher in the inflamed adipose tissue of women with greater hepatic fat content [3].

Ceramides act as second messengers in numerous signalling pathways involved in insulin action, inflammation, angiogenesis and cell death $[7,8]$. Ceramides are increased in serum, skeletal muscle and liver of obese rodents and humans (reviewed in [9]), correlate negatively with insulin sensitivity [10-12] and positively with circulating IL6 [13] thereby implicating ceramides in the development of insulin resistance and inflammation. Indeed, it was recently shown that a lowering of cellular ceramide mediates adiponectin's beneficial metabolic effects [12] and that ceramide links lipid-induced inflammatory pathways to the development of insulin resistance [14]. Data on adipose tissue ceramides, which are more limited and almost entirely restricted to rodent models, link increased ceramides or ceramide derivatives in this tissue to obesity, insulin resistance and local inflammation [15-20]. Therefore in the current study we investigate expression and localisation in human adipose tissue of enzymes involved in ceramide metabolism, with a particular focus on sphingomyelinases, to understand better the mechanisms underlying ceramide production in human adipose tissue since this is a largely unexplored area.

\section{Methods}

\section{Study populations}

Subcutaneous adipose tissue biopsies were collected from 20 obese, but otherwise healthy women (age 30-59 years, BMI $30-42 \mathrm{~kg} / \mathrm{m}^{2}$ ), recruited at Helsinki University Central Hospital, Finland [3]. The women were divided into two groups according to their liver fat content as measured by magnetic resonance proton spectroscopy: normal liver fat $(n=10$, liver fat range $1-3.5 \%)$ and high liver fat $(n=10$, range $6-35 \%)$. These groups were matched for age, BMI and subcutaneous and intra-abdominal fat masses as determined by MRI. However, compared to the normal liver fat group, the high liver fat group had higher concentrations of ceramide and sphingomyelin in subcutaneous adipose tissue and this tissue was more inflamed, as assessed by significantly higher expression levels of CD68, CCL2, CCL3 and PAI-1, and significantly lower expression levels of adiponectin and PPARG [3].

A second group consisted of 23 non-obese patients undergoing heart-valve surgery at Karolinska University Hospital, Stockholm, Sweden, without documented coronary artery disease. Biopsies were obtained at surgery from subcutaneous and intra-abdominal adipose tissue, and from the liver. Blood samples were taken after overnight fast. Clinical characteristics are summarized in Table 1. In addition, subcutaneous and intra-abdominal biopsies were taken at surgery from 8 morbidly obese patients ( 2 women, 6 men, age $45 \pm 2$ years, BMI $\left.52.6 \pm 2.0 \mathrm{~kg} / \mathrm{m}^{2}\right)$ undergoing laparoscopic gastric bypass operation at Helsinki University Central Hospital.

The nature and potential risks of the study were explained to all subjects before obtaining written informed consent. Study protocols were approved by the ethics committees of Karolinska Institutet and Helsinki University Central Hospital.

\section{Adipose tissue biopsies}

Tissue for gene expression analysis (approximately $150 \mathrm{mg}$ ) was either frozen in liquid nitrogen and kept at $-80^{\circ} \mathrm{C}$ prior to RNA extraction, for biopsies taken from obese subjects in Helsinki, or fixed in RNA-later (Ambion), for biopsies taken from patients undergoing heart-valve surgery in Stockholm. A part of each biopsy (from the 20 obese women and the 23 patients undergoing heart-valve surgery) was fixed in $4 \%$ zinc

Table 1 Clinical characteristics of patients undergoing heart-valve surgery

\begin{tabular}{lcc}
\hline & Males & Females \\
\cline { 2 - 3 } & $\mathbf{n}=\mathbf{1 4}$ & $\mathbf{n = 9}$ \\
\hline Age (years) & $64.8 \pm 3.9$ & $68.8 \pm 3.3$ \\
\hline BMl $\left(\mathrm{kg} / \mathrm{m}^{2}\right)$ & $27.2 \pm 1.0$ & $26.5 \pm 1.3$ \\
\hline Glucose $(\mathrm{mmol} / \mathrm{l})$ & $5.0 \pm 0.1$ & $5.2 \pm 0.2$ \\
\hline HbA1c $(\%)$ & $4.4 \pm 0.1$ & $4.7 \pm 0.1^{*}$ \\
\hline Insulin $(\mathrm{pmol} / \mathrm{l})$ & $56.2 \pm 10.5$ & $49.6 \pm 8.6$ \\
\hline Triacylglycerol $(\mathrm{mmol} / \mathrm{l})$ & $1.2 \pm 0.2$ & $0.9 \pm 0.1$ \\
\hline Total-cholesterol $(\mathrm{mmol} / \mathrm{l})$ & $5.1 \pm 0.2$ & $4.7 \pm 0.4$ \\
\hline LDL-cholesterol $(\mathrm{mmol} / \mathrm{l})$ & $3.4 \pm 0.2$ & $2.7 \pm 0.3$ \\
\hline HDL-cholesterol $(\mathrm{mmol} / \mathrm{l})$ & $1.2 \pm 0.1$ & $1.6 \pm 0.2^{*}$ \\
\hline ALAT $(\mu \mathrm{kat} / \mathrm{l})$ & $0.50 \pm 0.04$ & $0.41 \pm 0.04$ \\
\hline YGT $(\mu \mathrm{kat} / \mathrm{l})$ & $0.45 \pm 0.11$ & $0.48 \pm 0.20$ \\
\hline CRP $(\mathrm{mg} / \mathrm{l})$ & $2.9 \pm 0.6$ & $2.6 \pm 1.0$ \\
\hline
\end{tabular}

* $\mathrm{P}<0.05$ for differences between the groups. Values are expressed as mean \pm SEM. ALAT: alanine aminotransferase; $\gamma G T$ : gamma-glutamyl transferase; CRP: C-reactive protein. 
formaldehyde for subsequent paraffin embedding and immunohistochemistry. Tissue from the 20 obese women that had been frozen in liquid nitrogen and preserved at $-80^{\circ} \mathrm{C}$ was used for lipidomic analysis, as described previously [3]. In brief, adipose tissue lipids were extracted using chloroform:methanol (2:1) solvent and analysed using Ultra Performance Liquid Chromatography coupled to time-of-flight mass spectrometry (UPLC-QTOFMS). A total of 154 molecular lipids were measured including the following sphingolipids: ceramides $\operatorname{Cer}(\mathrm{d} 18: 0 / 22: 0)$, Cer (d18:1/16:0) and Cer(d18:1/24:1); and sphingomyelins SM (d18:1/16:0), SM(d18:1/18:0), SM(d18:1/20:0), SM(d18:1/ 22:0), SM(d18:1/22:1), SM(d18:1/24:1) and SM(d18:1/ $24: 2)$. All the identified lipids were quantified by calibrating with corresponding class-specific internal standards. Sphingomyelins were calibrated with phosphatidylcholine PC(17:0/17:0).

\section{RNA isolation and CDNA synthesis}

For biopsies collected in Sweden, approximately $150 \mathrm{mg}$ of adipose tissue was homogenized in Trizol (Invitrogen) using the Fastprep Homogenizer (Qbiogene). RNA purification, including DNase treatment, was performed using RNeasy mini kits (Qiagen) according to the manufacturer's protocol. RNA concentrations were measured using a NanoDrop spectrophotometer (Thermo). RNA quality was analyzed by an Agilent Bioanalyzer 2100 (Agilent Technologies). Isolated RNA was stored at $-80^{\circ} \mathrm{C}$ until cDNA synthesis. A total of $1 \mu \mathrm{g}$ RNA was transcribed into cDNA using Superscript III reverse transcriptase (Invitrogen) and oligo $(\mathrm{dT})_{12-18}$ primer. RNA isolation and CDNA synthesis from biopsies taken in Finland was described previously [3].

\section{Quantification of gene expression}

mRNA expression of specific genes was quantified by real time PCR using the ABI 7000 Sequence Detection System instrument and software (Applied Biosystems). cDNA synthesized from $15 \mathrm{ng}$ of total RNA was mixed with TaqMan Universal PCR Master Mix (Applied Biosystems) and a gene-specific primer and probe mixture (pre-developed TaqMan Gene Expression Assays, Applied Biosystems) in a final volume of $25 \mu \mathrm{l}$. The assays used were: Hs00154355_m1 for CD68, Hs00234140_m1 for CCL2, Hs00234142_m1 for CCL3, Hs00605917_m1 for adiponectin, Hs00174131_m1 for IL6, Hs00174128_m1 for TNFo, Hs99999910_m1 for TBP, Hs99999902_m1 for RPLP0 and assays shown in Table 2. All samples were run in duplicate. Relative expression levels were determined using a 5-point serially diluted standard curve, generated from cDNA from human adipose tissue. Gene expression was expressed in arbitrary units and normalized relative to the housekeeping genes RPLP0 and TBP to compensate for differences in cDNA loading. The average of these two values was used for normalization.

\section{Liver biopsies}

RNA extraction, gene expression analysis with Affymetrix GeneChip Human Exon 1.0 ST arrays and normalisation were performed as described [21]. Of the 23 patients undergoing heart-valve surgery included in the present study, liver data were available from 21. Hepatic expression levels of ACSL4, DGAT2, PNPLA3 and PPARG were selected for subsequent analysis.

\section{Immunohistochemistry}

Five $\mu \mathrm{m}$ thick serial sections were deparaffinized and boiled in TE-buffer (10 mM Tris-Cl, $1 \mathrm{mM}$ EDTA, $\mathrm{pH}$ $7.5)$ at $98^{\circ} \mathrm{C}$ for 30 min to demask epitopes. After blocking with normal serum (goat or horse, Vector Laboratories), sections were incubated with primary antibodies at $4^{\circ} \mathrm{C}$ overnight. Primary antibodies were: anti-SMPD1 sc-9815, 1:50, anti-SMPD2 sc-26212, 1:50, anti-SMPD3 sc-67692, 1:50, anti-PECAM-1 sc-1506, 1:100 (all goat polyclonal IgG, Santa Cruz Biotechnology) and mouse monoclonal anti-CD68, 1:200 (Novocastra Laboratories), anti-ASAH1, 1:50 (Abcam) and anti-apolipoprotein B, 1:50 (H61428M Biosite). After washing in PBS buffer, sections were incubated with secondary biotinylated goat anti mouse, 1:2000 (Dako) or biotinylated horse anti goat, 1:2000 (Vector Laboratories) antibodies. Staining was visualized using avidin-biotin peroxidase complex (ABC, Vector Laboratories) followed by 3.3'-diaminobenzidine tetrachloride (DAB, Vector Laboratories). All sections were counterstained with Harris haematoxylin (Histolab). Collagen was stained with Sirius red (Bie \& Berntsen) and visualized by polar light microscopy.

\section{Statistical analysis}

The Statview software (SAS Institute Inc, Cary, NC, USA) was used. Physical and biochemical characteristics of the study subjects were analyzed using nonparametric methods. Groups were compared using the Mann-Whitney test. Different adipose tissue depots were compared using the Wilcoxon signed rank test. All correlations were performed using Spearman's rank correlation. Statistical significance was assigned to a value of $P<0.05$. Data are presented as mean \pm SEM. Hepatic expression levels of selected genes were used to generate a mean standard deviation score for hepatic triacylglycerol accumulation. For each subject, each value was expressed as standard deviations of difference from the population mean. The mean hepatic triacylglycerol accumulation score was calculated as the mean of these standard deviation scores: (ACSL4+DGAT2 + PNPLA3 + PPARG) $/ 4$. 
Table 2 Expression in subcutaneous adipose tissue of ceramide-metabolising enzymes in relation to adipose tissue inflammation

\begin{tabular}{|c|c|c|c|c|c|}
\hline Gene & ABI assay ID & $\begin{array}{c}\mathrm{Ct} \\
\text { value }\end{array}$ & $\begin{array}{c}\text { Less } \\
\text { inflamed } \\
\text { adipose } \\
\text { tissue, } \\
\text { normal } \\
\text { liver fat }\end{array}$ & $\begin{array}{c}\text { More } \\
\text { inflamed } \\
\text { adipose } \\
\text { tissue, } \\
\text { high } \\
\text { liver fat }\end{array}$ & $P$ \\
\hline & & & $n=10$ & $n=10$ & \\
\hline SPTLC1 & Hs00272311_m1 & 27.2 & $0.99 \pm 0.04$ & $1.05 \pm 0.06$ & 0.20 \\
\hline SPTLC2 & Hs00191585_m1 & 26.8 & $0.97 \pm 0.07$ & $1.00 \pm 0.05$ & 0.39 \\
\hline DEGS1 & Hs00186447_m1 & 25.9 & $0.99 \pm 0.05$ & $1.07 \pm 0.05$ & 0.11 \\
\hline LASS1 & Hs00242151_m1 & 33.2 & $9.07 \pm 3.12$ & $9.22 \pm 1.63$ & 0.48 \\
\hline LASS4 & Hs00226114_m1 & 27.1 & $1.08 \pm 0.10$ & $1.07 \pm 0.16$ & 0.49 \\
\hline LASS6 & Hs00826756_m1 & 28.8 & $0.87 \pm 0.07$ & $0.89 \pm 0.07$ & 0.42 \\
\hline ASAH1 & Hs00602774_m1 & 24.3 & $1.16 \pm 0.10$ & $1.47 \pm 0.13$ & 0.03 \\
\hline UGCG & Hs00234293_m1 & 28.2 & $0.91 \pm 0.08$ & $0.96 \pm 0.06$ & 0.34 \\
\hline SGMS1 & Hs00380453_m1 & 28.0 & $1.01 \pm 0.05$ & $1.10 \pm 0.06$ & 0.12 \\
\hline SGMS2 & Hs00398067_m1 & 30.6 & $2.09 \pm 0.27$ & $2.57 \pm 0.32$ & 0.13 \\
\hline SMPD1 & Hs00609415_m1 & 26.6 & $1.51 \pm 0.10$ & $1.85 \pm 0.10$ & $0.01^{a}$ \\
\hline SMPD2 & Hs00162006_m1 & 29.8 & $1.13 \pm 0.06$ & $1.26 \pm 0.06$ & $0.08^{a}$ \\
\hline SMPD3 & Hs00218713_m1 & 33.8 & $1.33 \pm 0.13$ & $1.76 \pm 0.18$ & $0.05^{2}$ \\
\hline SMPD4 & Hs00215775_m1 & 29.1 & $1.57 \pm 0.09$ & $1.52 \pm 0.09$ & 0.35 \\
\hline CERK & Hs00368483_m1 & 29.3 & $1.57 \pm 0.15$ & $1.87 \pm 0.10$ & 0.06 \\
\hline SPHK1 & Hs00184211_m1 & 28.9 & $12.18 \pm 1.49$ & $17.64 \pm 1.65$ & 0.01 \\
\hline CGT & Hs00409961_m1 & 35.5 & $4.45 \pm 1.29$ & $3.45 \pm 0.52$ & 0.24 \\
\hline $\mathrm{CDH} 5$ & Hs00174344_m1 & 28.3 & $1.16 \pm 0.14$ & $1.43 \pm 0.13$ & 0.09 \\
\hline SELE & Hs00950401_m1 & 33.2 & $0.72 \pm 0.15$ & $2.45 \pm 0.94$ & 0.04 \\
\hline VEGF & Hs0090054_m1 & 26.4 & $4.13 \pm 0.37$ & $3.71 \pm 0.52$ & 0.25 \\
\hline HIF-1a & Hs00153153_m1 & 27.6 & $1.39 \pm 0.12$ & $1.65 \pm 0.08$ & 0.02 \\
\hline \multicolumn{6}{|c|}{ 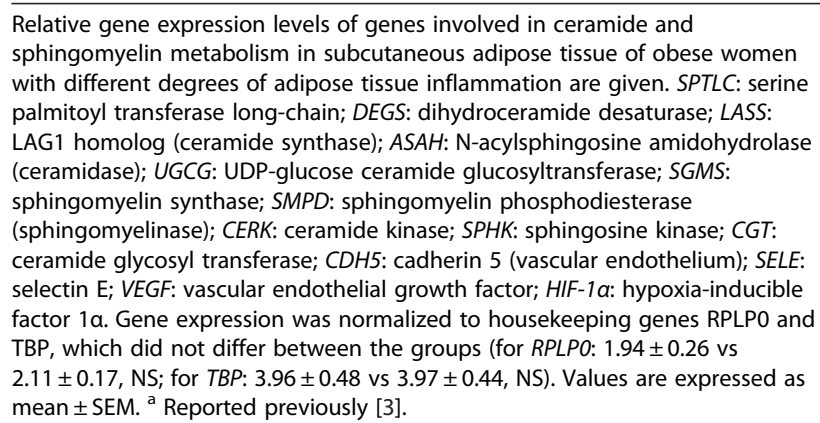 } \\
\hline
\end{tabular}

\section{Results}

To investigate the basis for elevated ceramide/sphingomyelin concentrations in inflamed adipose tissue, expression levels of genes involved in ceramide synthesis and metabolism (summarised in Figure 1) were quantified in subcutaneous adipose tissue from obese women. These women were divided into two groups based on their liver fat content: normal $(2.3 \pm 0.3 \%, \mathrm{n}=10)$ and high $(14.4 \pm 2.9 \%, \mathrm{n}=10)$. The groups were similar with respect to age, BMI and subcutaneous and intraabdominal adipose tissue masses, but the high liver fat group had a more unfavourable metabolic profile and more inflammation and higher ceramide and sphingomyelin concentrations in their subcutaneous adipose tissue [3]. Genes involved in de novo ceramide synthesis (SPTLC1, SPTLC2, DEGS1, LASS1, LASS4 and LASS6) were not differentially expressed between the groups (Table 2). Ceramide-metabolising enzymes CGT, CERK, SGMS1, SGMS2 and UGCG were also similarly expressed. However, ASAH1 and SPHK1 were expressed at significantly higher levels in the women with more inflamed adipose tissue $(P=0.03$ and $P=0.01$, respectively). Expression levels of three sphingomyelinases were greater $(P=0.01$ for SMPD1, $P=0.05$ for SMPD3), or tended to be greater $(P=0.08$ for SMPD2) in the women with high liver fat and more inflammation and higher ceramide/sphingomyelin content in their adipose tissue [3], but expression of a fourth sphingomyelinase, SMPD4, did not differ.

To establish whether ceramide and sphingomyelin metabolising enzymes that displayed differential mRNA expression between the two groups, namely SMPD1, SMPD2, SMPD3, ASAH1 and SPHK1, might be determinants of ceramide and/or sphingomyelin concentrations in adipose tissue, their mRNA levels were correlated with these lipid species (Table 3). Expression levels of SMPD3 in adipose tissue were statistically significantly correlated with 2 out of the 3 ceramides and 6 out of the 7 sphingomyelins that were quantified with the lipidomic analysis. SPHK1 expression was significantly correlated with one ceramide and two sphingomyelin species.

Collectively these data suggested a possible role for sphingomyelinase activity in adipose tissue in determining adipose ceramide and/or sphingomyelin concentrations, which may be related to inflammation and macrophage accumulation. To establish the location of these enzymes in adipose tissue, immunohistochemical analysis of subcutaneous adipose tissue from the obese women was performed. SMPD1-3 proteins were expressed in both macrophages and adipocytes within adipose tissue (Figure 2). All three sphingomyelinases were also found in and around blood vessels (Figure 2F-H).

To investigate sphingomyelinases further in human adipose tissue, the mRNA expression and protein distribution of SMDP1-3 was compared in subcutaneous and intra-abdominal adipose tissue from non-obese individuals (clinical characteristics in Table 1). Gene expression levels of the inflammatory markers CD68, CCL2 and IL6 were significantly higher in intra-abdominal compared to subcutaneous fat (Figure 3A). SMPD3 mRNA was higher, while expression of SMPD2 was lower in intra-abdominal compared to subcutaneous fat. Expression of SMPD1 was similar in both depots. Immunohistochemical analysis of subcutaneous and 


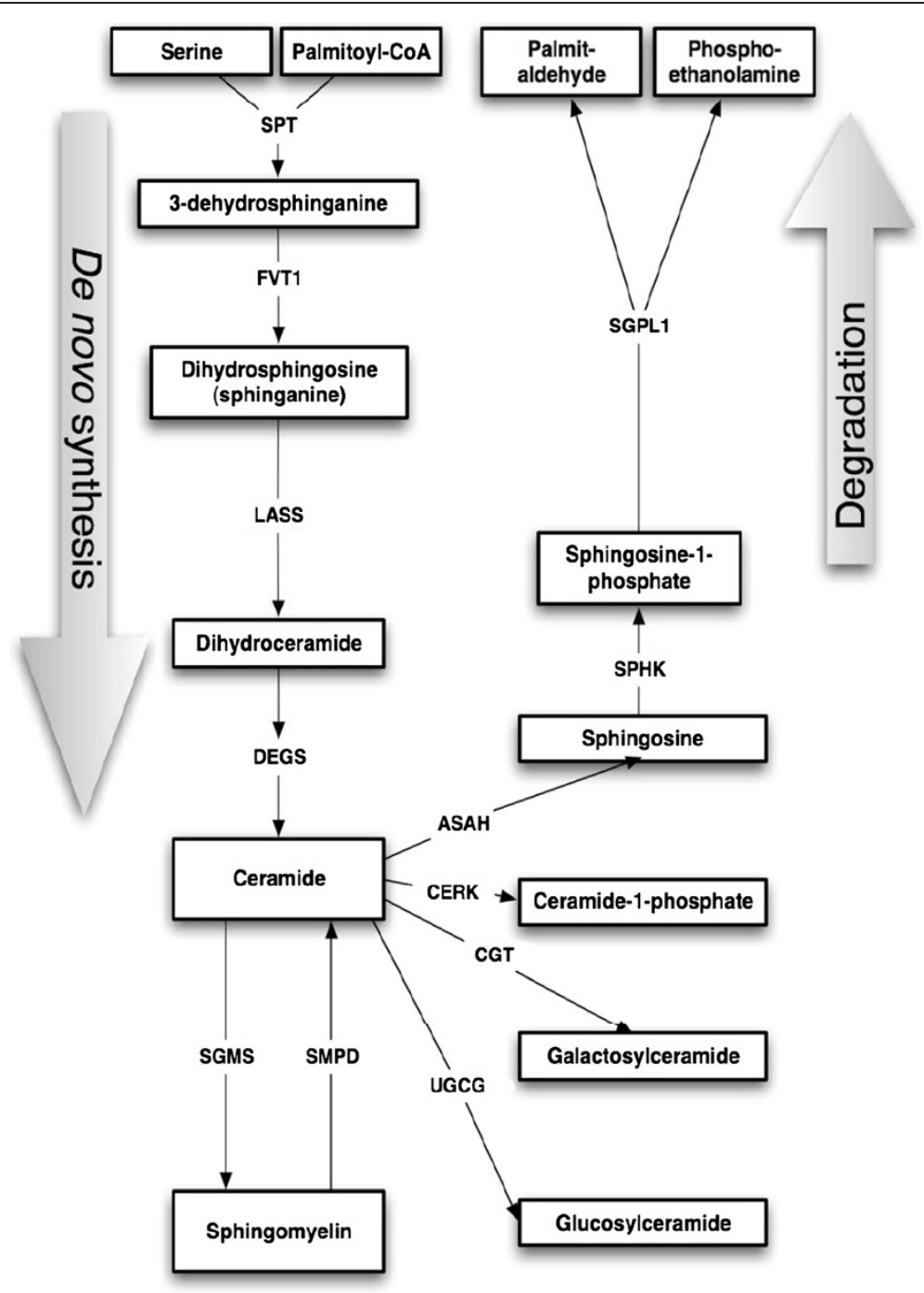

Figure 1 Schematic diagram depicting enzymatic reactions involved in the synthesis and degradation of ceramide and sphingomyelin.

intra-abdominal adipose tissue located SMPD1-3 proteins mainly to blood vessels (Figure $4 \mathrm{~A}$ and $\mathrm{C}$ for SMPD3). Furthermore, an enzyme that metabolises ceramides, ASAH1, was also localized to the vasculature (Figure 4E). In light of our previous data from obese women identifying a relationship between inflammation and ceramide metabolism in adipose tissue and fat accumulation in the liver, we investigated associations between gene expression in adipose tissue and liver in these non-obese individuals. Since direct measurements of liver fat were not available, hepatic mRNA levels of selected genes were used as surrogate markers of liver fat accumulation. Due to the small sample size, only a very limited number of genes were selected: ACSL4, DGAT2, PNPLA3 and PPARG. We have previously shown hepatic expression of ACSL4 and PPARG to be related to liver fat content [22]. DGAT catalyses the committed step in triacylglycerol synthesis with DGAT2 being the dominant DGAT enzyme controlling triacylglycerol homeostasis in vivo [23], and PNPLA3 has been implicated in the development of hepatic steatosis [24]. A mean standard deviation score was calculated from the hepatic mRNA expression data for these 4 genes (see methods) to generate a summary score for liver triacylglycerol accumulation. This liver summary score was significantly correlated to expression levels of CD68 (chosen as a marker of macrophage accumulation) in both subcutaneous and intra-abdominal adipose tissue $(\mathrm{r}=0.56, P=0.01$ and $r=0.49 P=0.03$ respectively), but not to adipose tissue sphingomyelinase expression in either depot (SMPD1: $\mathrm{r}=0.10, P=0.65$ and $\mathrm{r}=0.24, P=0.29 ;$ SMPD2: $\mathrm{r}=0.16$, $P=0.48$ and $\mathrm{r}=0.33, P=0.14 ;$ and SMPD3: $\mathrm{r}=0.10$, $P=0.65$ and $\mathrm{r}=0.21, P=0.36$ for subcutaneous and intraabdominal adipose tissue respectively). 
Table 3 Correlations between sphingomyelinase, ceramidase and sphingosine kinase gene expression, and ceramide and sphingomyelin concentrations in subcutaneous adipose tissue

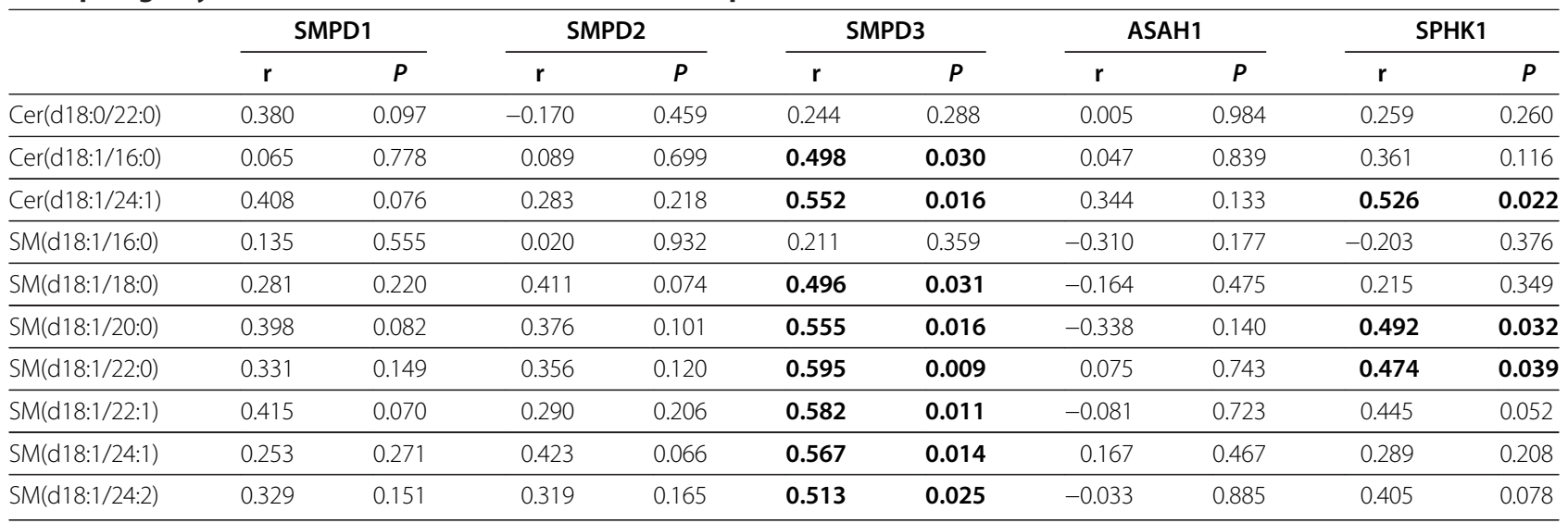

Correlations between gene expression levels of SMPD1-3 (sphingomyelinases), ASAH1 (ceramidase) and SPHK1 (sphingosine kinase) and concentrations of various ceramides (cer) and sphingomyelins (SM) in subcutaneous adipose tissue of obese women $(n=20)$. Spearman rank correlation coefficients and significance levels are shown. Comparison of sphingolipid concentrations (mean $\pm \mathrm{SEM}, \mathrm{nmol} / \mathrm{g}$ adipose tissue) in subcutaneous adipose tissue from women with less inflamed adipose tissue/normal liver fat $(n=10)$ versus more inflamed adipose tissue/high liver fat content $(n=10)$, as reported previously [3]: Cer(d18:0/22:0): $3.80 \pm 0.58$ vs $6.21 \pm 1.11, P=0.076 ; \operatorname{Cer}(d 18: 1 / 16: 0): 2.66 \pm 0.28$ vs $3.12 \pm 0.23, P=0.219 ; \operatorname{Cer}(d 18: 1 / 24: 1): 5.36 \pm 0.47$ vs $7.97 \pm 0.70, P=0.006 ; S M(d 18: 1 / 16: 0): 36.86 \pm 2.64$ vs $35.10 \pm 2.64, P=0.643 ; \mathrm{SM}(\mathrm{d} 18: 1 / 18: 0): 4.88 \pm 0.40$ vs $6.02 \pm 0.46, P=0.077 ; \mathrm{SM}(\mathrm{d} 18: 1 / 20: 0): 5.84 \pm 0.50$ vs $7.30 \pm 0.52, P=0.055 ; \mathrm{SM}(\mathrm{d} 18: 1 / 22: 0): 11.38 \pm 0.96$ vs $15.38 \pm 1.15, P=0.015 ; \mathrm{SM}(\mathrm{d} 18: 1 / 22: 1): 7.13 \pm 0.54$ vs $8.54 \pm 0.67, P=0.121 ; \mathrm{SM}(\mathrm{d} 18: 1 / 24: 1): 17.00 \pm 1.33$ vs $22.96 \pm 1.70, P=0.013 ; \mathrm{SM}(\mathrm{d} 18: 1 / 24: 2): 7.42 \pm 0.56$ vs $9.69 \pm 0.76, P=0.028$. Lipidomic analysis was described previously [3].

Expression patterns of SMPD1-3 mRNA in subcutaneous and intra-abdominal adipose tissue were also determined in an independent group of 8 morbidly obese subjects (Figure 3B). Levels of SMPD3 mRNA were significantly greater in intra-abdominal than in subcutaneous adipose tissue, but no significant differences were observed for SMPD1 or SMPD2. Differences in gene expression between adipose tissue depots could not be explained by depot-specific differences in housekeeping gene expression and essentially identical results were obtained when non-normalised gene expression data (raw Ct values) were used (data not shown).

Since staining for sphingomyelinases in adipose tissue was strongest in the vicinity of blood vessels, we investigated whether there were differences in adipose tissue vascularity between women with more or less inflammation in their adipose tissue. However, expression levels of a marker of vascular endothelium (CDH5) did not differ between groups (Table 2). Expression levels of markers of hypoxia (HIF-1 $\alpha$ ) and endothelial activation (E-selectin) were significantly higher in the adipose tissue of women with an increased liver fat content and more inflammation and higher ceramide concentrations in their adipose tissue (Table 2), but these increases in hypoxia and endothelial activation did not appear to be associated with an increase in angiogenesis, since there was no corresponding increase in expression of the marker for endothelial growth, VEGF. In adipose tissue from both obese and non-obese subjects, staining for the integral protein of hepatic lipoproteins, apolipoprotein B (apo B), was found in blood vessels, but also in regions that stained positive for CD68 (Figure 5), suggesting that lipoproteins entering adipose tissue via the vasculature might provide a source of sphingomyelins for hydrolysis by local sphingomyelinases to produce ceramide.

\section{Discussion}

We investigated sphingolipid metabolism in human adipose tissue to identify pathways underlying increased ceramide concentrations in inflamed adipose tissue [3]. Our data suggest that hydrolysis of sphingomyelin to ceramide by sphingomyelinases could explain, at least partly, this increase. Gene expression levels of SMPD3 correlate significantly with concentrations of various ceramides and sphingomyelins in subcutaneous adipose tissue, and are higher in the relatively more inflamed intra-abdominal compared to the subcutaneous depot in both obese and non-obese subjects. Sphingomyelinases are expressed by both adipocytes and macrophages in adipose tissue, but their expression is strongest in and around blood vessels. Our findings implicate a role for sphingomyelinase-mediated generation of ceramide in adipose tissue inflammation.

When comparing inflamed ceramide-rich and relatively less inflamed ceramide-poor subcutaneous adipose tissue of obese women, we report here for the first time that there were no differences in mRNA levels of genes involved in de novo ceramide synthesis. However, expression of sphingomyelinases SMPD1 and SMPD3 was significantly higher, while that of SMPD2 tended to be higher in the inflamed adipose tissue group (as reported [3]). Since sphingomyelinases catalyse the conversion of 


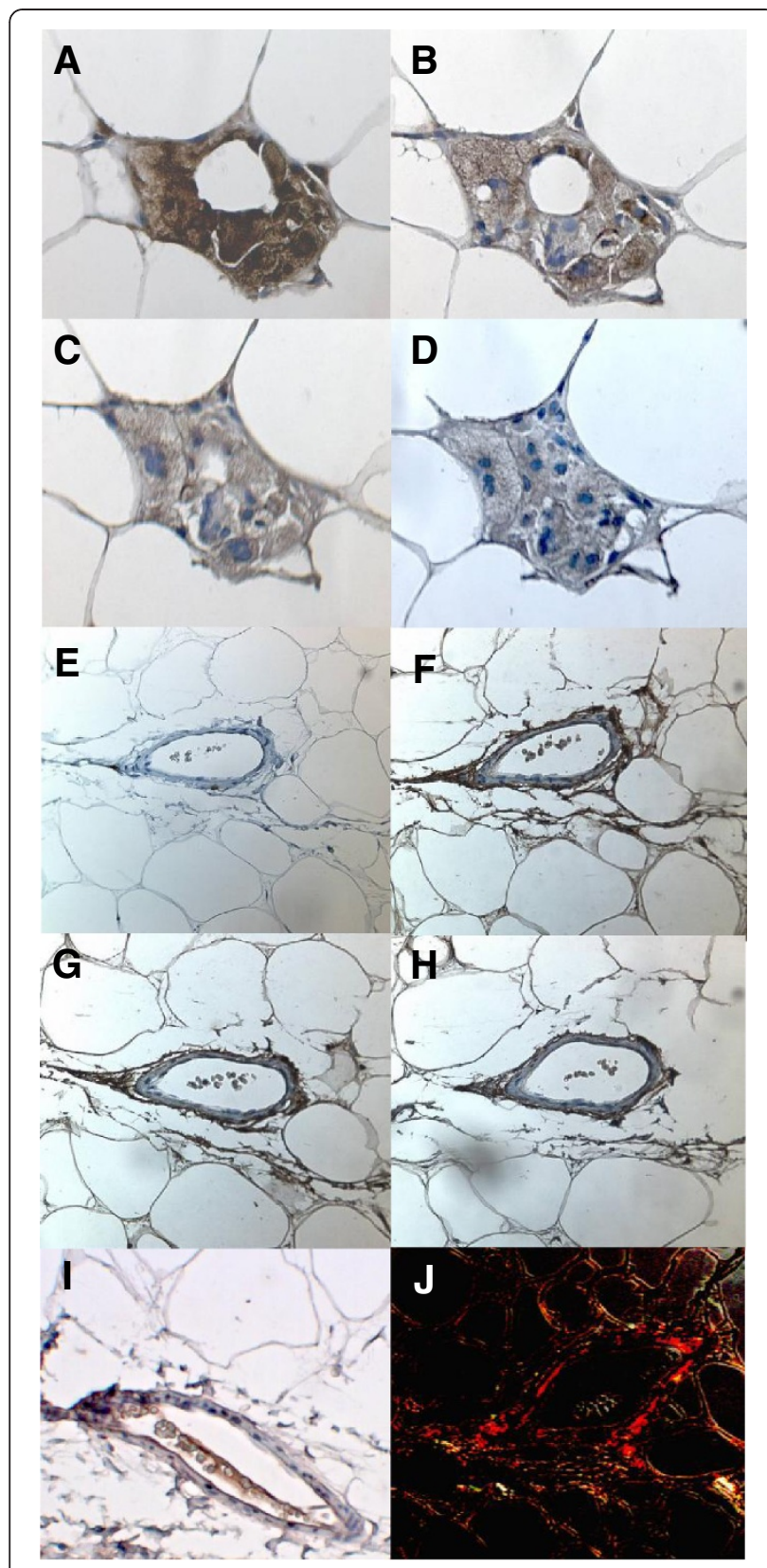

Figure 2 Localisation of sphingomyelinases in subcutaneous adipose tissue. Representative pictures of immunohistochemical staining of macrophages and multinuclear giant cells (aggregated macrophages) (A-D) and a small blood vessel (E-J) within subcutaneous adipose tissue from an obese woman. Brown coloration indicates macrophage-specific CD68 (A and E), SMPD1 (B and $\mathbf{F}$ ), SMPD2 (C and $\mathbf{G}$ ), SMPD3 (D and $\mathbf{H}$ ) and PECAM-1 (I). All sections were counterstained with haematoxylin (coloured blue). Collagen staining (J) was visualized with polarized light to reveal mature, extracellular collagen (coloured red).

sphingomyelins to ceramide, this pathway rather than de novo ceramide synthesis, may underlie the increased ceramide content of the inflamed adipose tissue of these women. Sphingomyelinase activity is increased by oxidative stress both in vitro [25] and in vivo [26] and sphingomyelinase expression in adipose tissue increases in response to a high fat diet in mouse models [15,27]. Since ceramides stimulate synthesis of pro-inflammatory cytokines by both adipocytes and macrophages [27,28], increased sphingomyelinase activity in adipose tissue could exacerbate the inflammatory milieu and enhance recruitment of macrophages. Therefore investigation of sphingomyelinases in human adipose tissue in relation to inflammation and macrophage accumulation is motivated. To date the only report of sphingomyelinases in human adipose tissue found reduced acid sphingomyelinase, but unchanged neutral sphingomyelinase activity in obese compared to lean patients [29].

Although we observed increased sphingomyelinase mRNA expression in inflamed adipose tissue, sphingomyelinases did not localise only to inflammatory cells (determined by immunohistochemistry). Staining for SMPD1, -2 and -3 was seen in macrophages and also in adipocytes, but the strongest staining was seen in and around blood vessels, the latter being reminiscent of the secretory form of SMPD1 localising to the subendothelial matrix of atherosclerotic lesions [30]. Additionally, the ceramide-metabolising enzyme ASAH1 was found in the vasculature, indicating that blood vessels are important sites for ceramide metabolism within adipose tissue. Indeed, immunohistochemical analysis revealed staining for apo B in areas containing inflammatory cells (positive for CD68) and within blood vessels, indicating access of adipose tissue sphingomyelinases to sphingomyelins within lipoproteins. Previously we found that not only ceramides, but also sphingomyelins were increased in adipose tissue of obese women with more inflamed adipose tissue. The increase in sphingomyelins did not appear to be accounted for by increased local synthesis (as discussed above), but might be explained by an increased delivery of sphingomyelin-rich lipoproteins produced by fatty liver [31], since these women also had increased hepatic fat content, but quantification of lipoprotein delivery to or retention within adipose tissue was not possible in this study. To pursue this idea we investigated the relationship between hepatic triacylglycerol accumulation and adipose tissue ceramide metabolism and inflammation in non-obese individuals. Inflammation in both subcutaneous and intra-abdominal adipose tissue (as assessed by RNA levels of the macrophage marker CD68) was positively related to the expression of genes in the liver reflecting triacylglycerol accumulation, but no such relationships were found for sphingomyelinase expression (SMPD1, -2 or -3 ) in either adipose tissue depot. This suggests that even in non-obese individuals, the number of macrophages within adipose tissue is linked to hepatic triacylglycerol metabolism. However, sphingomyelinases do not appear 


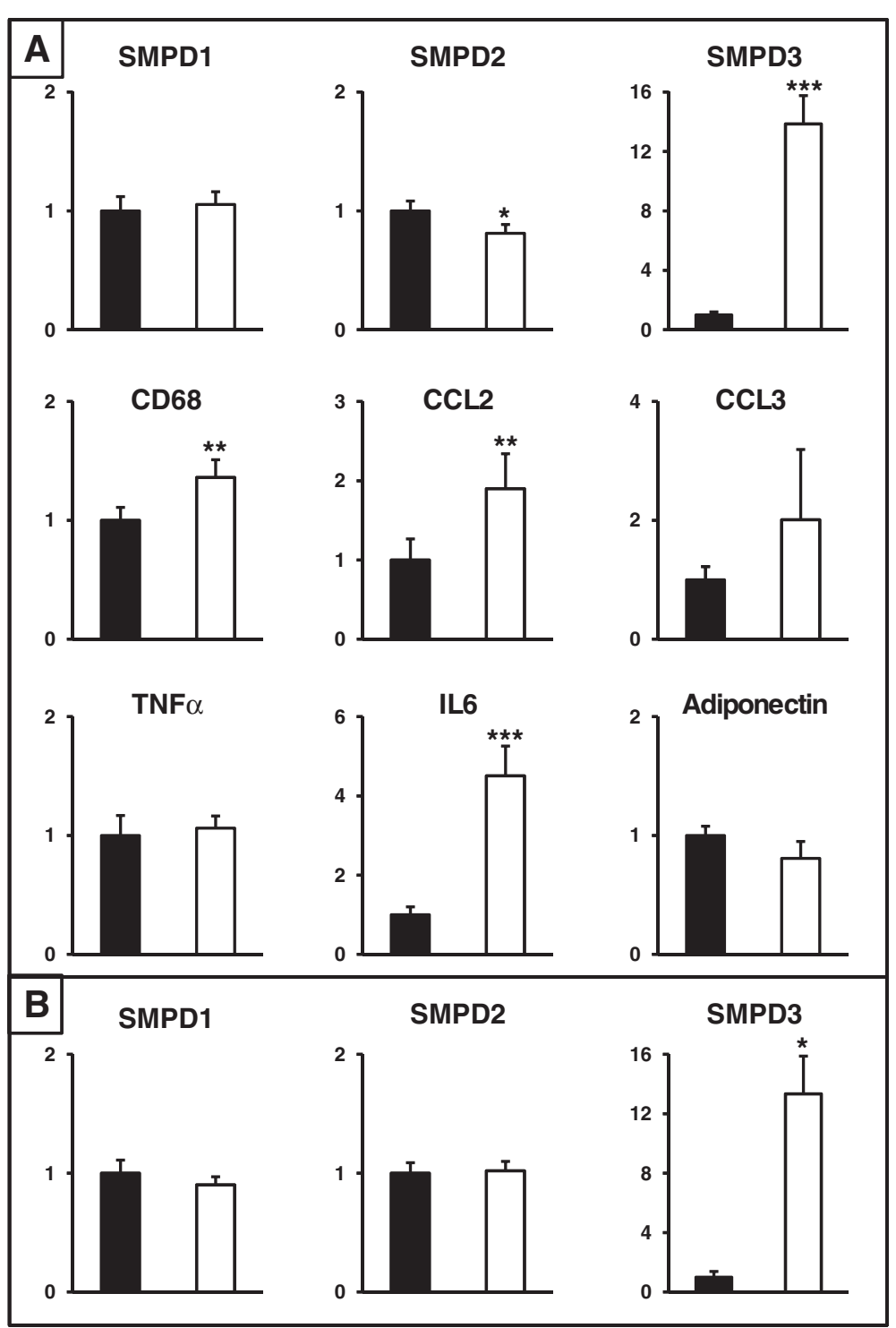

Figure 3 Gene expression in subcutaneous and intra-abdominal adipose tissue from non-obese and obese individuals. (A) Relative gene expression levels of SMPD1-3, CD68, CCL2, CCL3, TNFa, IL6 and adiponectin in subcutaneous (black bars) and intra-abdominal (white bars) adipose tissue from 23 non-obese individuals. (B) Relative gene expression levels of SMPD1-3 in subcutaneous (black bars) and intra-abdominal (white bars) adipose tissue from 8 obese patients. Expression is in arbitrary units normalized to housekeeping genes RPLP0 and TBP, and set to 1 for the subcutaneous depot. ${ }^{*} P<0.05,{ }^{* *} P<0.01,{ }^{* * *} P<0.001$ compared to subcutaneous adipose tissue.

to be involved in this relationship in these subjects. Unfortunately no measurements of either adipose tissue ceramide concentrations or liver fat content were available, so no conclusions can be drawn as to the relationship between adipose tissue macrophage accumulation, ceramide concentration and hepatic triacylglycerol content, but our data suggest that sphingomyelinase-mediated generation of ceramide in adipose tissue does not play a major role in this context in non-obese subjects who are unlikely to have fatty livers.

Our data highlighted the potential importance of SMPD3 within adipose tissue in relation to ceramide generation and inflammation for two reasons. Firstly, mRNA levels of SMPD3 correlated significantly with ceramide and sphingomyelin concentrations within adipose tissue of obese women. Secondly, a relatively more inflamed adipose tissue depot, namely intra-abdominal fat, expressed SMPD3 mRNA at significantly greater levels than relatively less inflamed subcutaneous adipose tissue in both non-obese and obese subjects. It is possible that increased SMPD3 activity contributes to the greater ceramide concentrations in intra-abdominal compared to subcutaneous adipose tissue [32]. Adipose tissue hypoxia is proposed to be a major underlying 


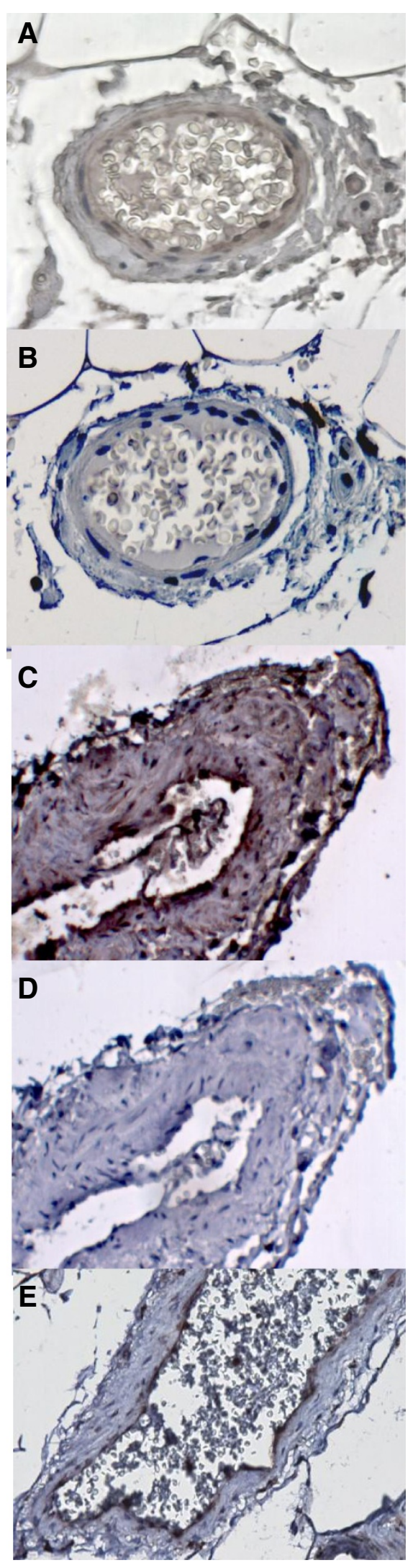

Figure 4 Localisation of sphingomyelinase SMPD3 and ceramidase ASAH1 in subcutaneous and intra-abdominal adipose tissue. Representative pictures of immunohistochemical staining of a vessel within subcutaneous (A, B and $\mathbf{E}$ ) and intraabdominal ( $\mathbf{C}$ and $\mathbf{D}$ ) adipose tissue from a non-obese individual. Positive staining for SMPD3 (A and $\mathbf{C}$ ) and ASAH1 (E) is shown as brown coloration. Negative staining is shown in panels $\mathbf{B}$ and $\mathbf{D}$. All sections were counterstained with haematoxylin (coloured blue).

cause for insulin resistance and other disorders associated with obesity, promoting macrophage infiltration and angiogenesis [33]. The location of sphingomyelinases to blood vessels within adipose tissue might indicate their involvement in angiogenesis. The increased expression of markers of hypoxia and endothelial activation, but no differences in markers of either angiogenesis or endothelial cell number in adipose tissue of women with inflamed compared to less inflamed adipose tissue, indicates the existence of hypoxia and an activated endothelium without an apparent decrease in vessel density or onset of angiogenesis. It is possible that the increased degree of inflammation is related to an inability of hypoxia and increased ceramide concentrations to induce angiogenesis within adipose tissue. Since particular importance was assigned to SMPD3 in hypoxic vasoconstriction in the lung [34], SMPD3 may play a role linking hypoxia, ceramide generation and inflammation within adipose tissue, however, this remains to be shown.

The observation that concentrations of some ceramide and sphingomyelin species correlated positively with gene expression levels of SPHK1 (sphingosine kinase) in adipose tissue in the cohort of obese women is also of interest. The product of SPHK1 action is sphingosine1-phosphate (concentrations of which were not quantified in the present study), a sphingolipid that appears to have the opposite actions to that of ceramide, namely promoting cell survival and proliferation [35]. Indeed, the balance between concentrations of ceramide and sphingosine-1-phosphate is proposed to be an important mechanism controlling cell fate [35]. One interpretation of our data could be that the higher expression levels of SPHK1 in the more inflamed adipose tissue of the women with fatty livers, as compared to the less inflamed adipose tissue of women with normal liver fat content, and the correlations between SPHK1 expression and ceramide/sphingomyelin concentrations in the cohort as a whole reflect a protective mechanism to counteract the potentially detrimental consequences of ceramide accumulation, but this is speculative and future studies are needed to investigate this.

Strengths of our study include the investigation of human adipose tissue biopsies from three independent patient groups and two different depots, and the availability of measures of hepatic fat content in the obese 


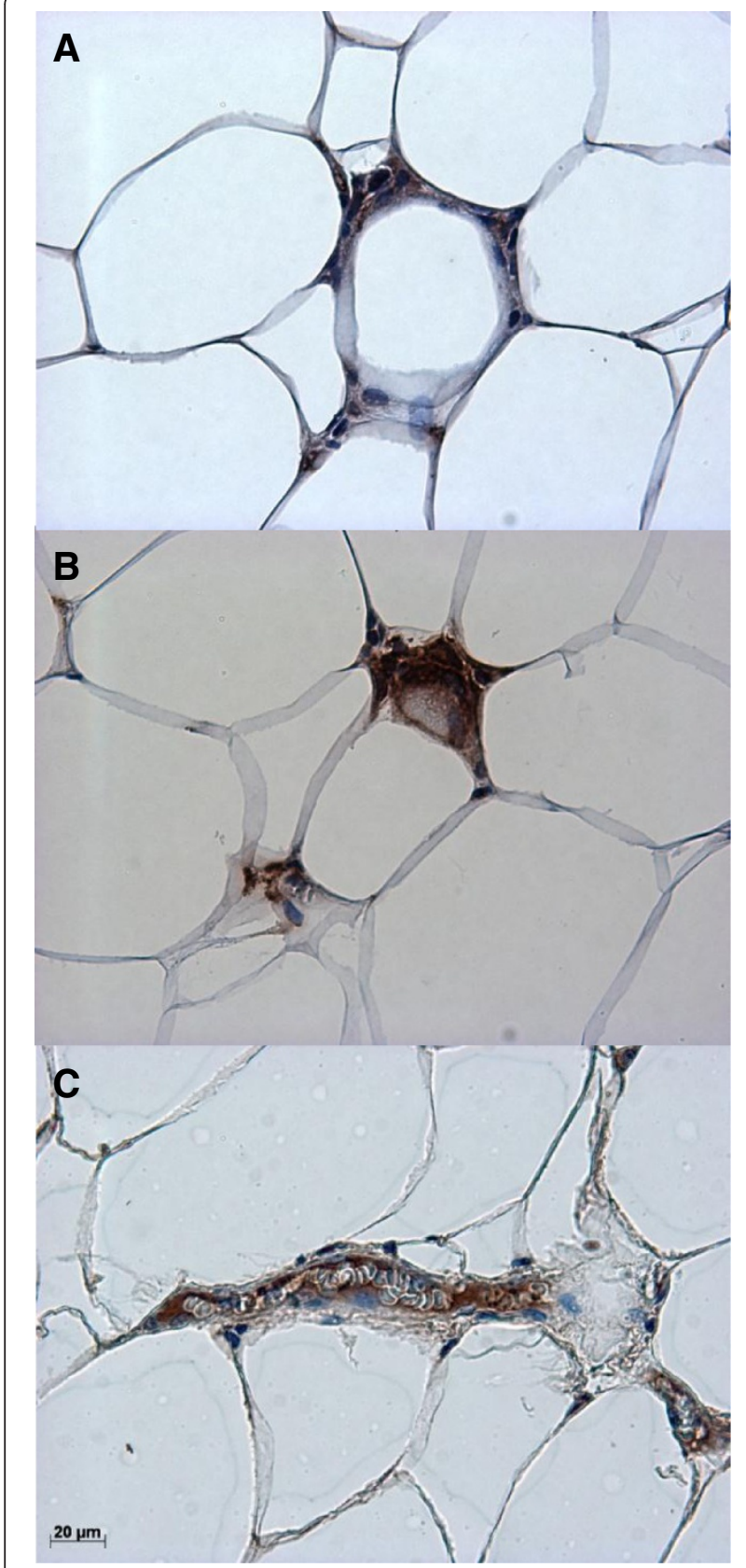

Figure 5 Localisation of apo B in subcutaneous and intraabdominal adipose tissue. Representative pictures of immunohistochemical staining of apo B (A, C) and CD68 (B) in human adipose tissue. (A) and (B): Serial sections of subcutaneous adipose tissue from an obese woman stained for apo B and CD68 respectively. (C): Intra abdominal adipose tissue from a non-obese individual stained for apo B. Positive staining for apo B (A and $\mathbf{C}$ ) and CD68 (B) is shown as brown coloration. All sections were counterstained with haematoxylin (coloured blue).

women, and hepatic gene expression data in the nonobese subjects enabling us to investigate relationships between liver and adipose tissue. A limitation is that comparisons between the different patient groups cannot be made since the groups were not anlaysed at the same time, thus only within group comparisons can be made. Another limitation is the availability of only mRNA quantification of sphingomyelinase expression rather than protein concentration or enzyme activity. Nonetheless, protein expression of sphingomyelinase was confirmed and its location in adipose tissue established by immunohistochemistry. The small size of the biopsies obtained precluded more extensive analysis. Analysis of only adipocytes was not performed since we aimed to explore the expression of ceramide-metabolising enzymes in adipose tissue as a whole (including inflammatory cells, connective tissue, blood vessels etc.) rather than exclusively in adipocytes. However, immunohistochemical analysis provided information as to the cellular location of certain proteins.

\section{Conclusions}

Our data suggest that sphingomyelinase-mediated production of ceramide from sphingomyelin may be one mechanism contributing to the development of inflammation within human adipose tissue. An increased expression of SMPD3 in inflamed adipose tissue and in intra-abdominal compared to subcutaneous tissue, and its close association with adipose tissue ceramide levels might indicate an important role for this sphingomyelinase.

\section{Abbreviations}

ACSL4: Acyl-CoA synthetase long-chain family member 4; ASAH: $\mathrm{N}$-acylsphingosine amidohydrolase (ceramidase); Apo B: Apolipoprotein B; CCL2: Chemokine (C-C motif) ligand 2 (monocyte chemoattractant protein 1 MCP-1); CCL3: Chemokine (C-C motif) ligand 3; CDH5: Cadherin 5 (vascular endothelium); CERK: Ceramide kinase; CGT: Ceramide glycosyl transferase; DEGS: Dihydroceramide desaturase; DGAT2: Diacylglycerol O-acyltransferase 2; HIF-1a: Hypoxia-inducible factor 1a; LASS: LAG1 homolog (ceramide synthase); PNPLA3: Patatin-like phospholipase domain-containing protein 3 (adiponutrin); PPARG: Peroxisome proliferator activated receptor gamma; RPLPO: Ribosomal protein large PO; SELE: Selectin E; SGMS: Sphingomyelin synthase; SMPD: Sphingomyelin phosphodiesterase (sphingomyelinase); SPTLC: Serine palmitoyl transferase long-chain; TBP: TATA box binding protein; UGCG: UDP-glucose ceramide glucosyltransferase; VEGF: Vascular endothelial growth factor.

\section{Competing interests}

The authors declare that they have no competing interests.

\section{Authors' contributions}

MK participated in study design, data collection, statistical analysis, data interpretation and manuscript writing. JG, JW, JL, AF-C and MO participated in data collection. SAS participated in study design. HY-J participated in study design and data interpretation. PE participated in study design, data interpretation and manuscript writing. RMF conceived the study, participated in study design, statistical analysis, data interpretation and manuscript writing. All authors read and approved the final manuscript.

\section{Acknowledgments}

This study was supported by the Swedish Research Council (project 15352), Novo Nordisk Foundation, Swedish Diabetes Association, Stockholm County Council (project 562183), the Network for Circulation and Respiration at Karolinska Institutet, Academy of Finland, Sigrid Juselius Foundation, Finnish Diabetes Research Foundation and a donation by Fredrik Lundberg. It is part of the project "Hepatic and adipose tissue and functions in the metabolic syndrome" (www.hepadip.org), which is supported by the European 
Commission as an Integrated Project under the 6th Framework Programme (Contract LSHM-CT-2005-018734). We acknowledge Mia Urjansson, Katja Tuominen and Laxman Yetukuri for excellent technical assistance.

\section{Author details}

'Atherosclerosis Research Unit, Department of Medicine (Solna), Center for Molecular Medicine, Karolinska Institutet, Stockholm, Sweden. ${ }^{2}$ Division of Diabetes, Department of Medicine, University of Helsinki, Helsinki, Finland. ${ }^{3}$ Program in Cardiovascular and Metabolic Diseases, Duke-National University of Singapore Graduate Medical School, Singapore. ${ }^{4}$ Cardiothoracic Surgery Unit, Department of Molecular Medicine and Surgery, Karolinska Institutet, Stockholm, Sweden. ${ }^{5}$ VTT Technical Research Centre of Finland, Espoo, Finland.

Received: 21 June 2012 Accepted: 5 September 2012 Published: 13 September 2012

\section{References}

1. Weisberg SP, McCann D, Desai M, Rosenbaum M, Leibel RL, Ferrante AW Jr. Obesity is associated with macrophage accumulation in adipose tissue. $J$ Clin Invest 2003, 112:1796-1808.

2. Cinti S, Mitchell G, Barbatelli G, Murano I, Ceresi E, Faloia E, Wang S, Fortier $M$, Greenberg AS, Obin MS: Adipocyte death defines macrophage localization and function in adipose tissue of obese mice and humans. J Lipid Res 2005, 46:2347-2355.

3. Kolak M, Westerbacka J, Velagapudi VR, Wågsäter D, Yetukuri L, Makkonen J, Rissanen A, Hakkinen AM, Lindell M, Bergholm R, et al: Adipose tissue inflammation and increased ceramide content characterize subjects with high liver fat content independent of obesity. Diabetes 2007, 56:1960-1968.

4. Goossens $\mathrm{GH}$ : The role of adipose tissue dysfunction in the pathogenesis of obesity-related insulin resistance. Physiol Behav 2008, 94:206-218.

5. Barbarroja N, Lopez-Pedrera R, Mayas MD, Garcia-Fuentes E, Garrido-Sanchez L, Macias-Gonzalez M, El Bekay R, Vidal-Puig A, Tinahones FJ: The obese healthy paradox: is inflammation the answer? Biochem J 2010, 430:141-149.

6. Yki-Jarvinen $\mathrm{H}$, Westerbacka J: The fatty liver and insulin resistance. Curr Mol Med 2005, 5:287-295.

7. Summers SA: Ceramides in insulin resistance and lipotoxicity. Prog Lipid Res 2006, 45:42-72.

8. Hannun YA, Obeid LM: Principles of bioactive lipid signalling: lessons from sphingolipids. Nat Rev Mol Cell Biol 2008, 9:139-150.

9. Holland WL, Summers SA: Sphingolipids, insulin resistance, and metabolic disease: new insights from in vivo manipulation of sphingolipid metabolism. Endocr Rev 2008, 29:381-402.

10. Haus JM, Kashyap SR, Kasumov T, Zhang R, Kelly KR, DeFronzo RA, Kirwan JP: Plasma ceramides are elevated in obese subjects with type 2 diabetes and correlate with the severity of insulin resistance. Diabetes 2009, 58:337-343.

11. Straczkowski M, Kowalska I, Baranowski M, Nikolajuk A, Otziomek E, Zabielski P, Adamska A, Blachnio A, Gorski J, Gorska M: Increased skeletal muscle ceramide level in men at risk of developing type 2 diabetes. Diabetologia 2007, 50:2366-2373.

12. Holland WL, Miller RA, Wang ZV, Sun K, Barth BM, Bui HH, Davis KE, Bikman BT, Halberg N, Rutkowski JM, et al: Receptor-mediated activation of ceramidase activity initiates the pleiotropic actions of adiponectin. Nature Med 2011, 17:55-63.

13. de Mello V, Lankinen M, Schwab U, Kolehmainen M, Lehto S, Seppänen-Laakso T, Orešic M, Pulkkinen L, Uusitupa M, Erkkilä AT: Link between plasma ceramides, inflammation and insulin resistance: association with serum IL-6 concentration in patients with coronary heart disease. Diabetologia 2009, 52:2612-2615.

14. Holland WL, Bikman BT, Wang L-P, Yuguang G, Sargent KM, Bulchand S, Knotts TA, Shui G, Clegg DJ, Wenk MR, et al: Lipid-induced insulin resistance mediated by the proinflammatory receptor TLR4 requires saturated fatty acid-induced ceramide biosynthesis in mice. J Clin Invest 2011, 121:1858-1870.

15. Shah C, Yang G, Lee I, Bielawski J, Hannun YA, Samad F: Protection from high fat diet-induced increase in ceramide in mice lacking plasminogen activator inhibitor 1. J Biol Chem 2008, 283:13538-13548.
16. Van Eijk M, Aten J, Ottenhoff R, Van Roomen CPAA, Dubbelhuis PF, Seeman I, Ghauharali-VanDer Vlugt K, Overkleeft HS, Arbeeny C, Groen AK, et al: Reducing glycosphingolipid content in adipose tissue of obese mice restores insulin sensitivity, adipogenesis and reduces inflammation. PLOS One 2009, 4:e4723.

17. Wu D, Ren Z, Pae M, Guo W, Cui X, Merrill AH, Nikbin Meydani S: Aging up-regulates expression of inflammatory mediators in mouse adipose tissue. J Immunol 2007, 179:4829-4839.

18. Bonzón-Kulichenko E, Schwudke D, Gallardo N, Moltó E, Fernández-Agulló T, Shevchenko A, Andrés A: Central leptin regulates total ceramide content and sterol regulatory element binding protein-1c proteolytic maturation in rat white adipose tissue. Endocrinology 2009, 150:169-178.

19. Yang G, Badeanlou L, Bielawski J, Roberts AJ, Hannun YA, Samad F: Central role of ceramide biosynthesis in body weight regulation, energy metabolism, and the metabolic syndrome. Am J Physiol Endocrinol Metab 2009, 297:E211-E224.

20. Blachnio-Zabielska AU, Koutsari C, Tchkonia T, Jensen MD: Sphingolipid content of human adipose tissue: relationship to adiponectin and insulin resistance. Obesity 2012, Epub 7 June 2012.

21. Folkersen L, Van't Hooft F, Chernogubova E, Agardh HE, Hansson GK, Hedin U, Liska J, Syvanen AC, Paulsson-Berne G, Franco-Cereceda A, et al: Association of genetic risk variants with expression of proximal genes identifies novel susceptibility genes for cardiovascular disease. Circ Cardiovasc Genet 2010, 3:365-373

22. Westerbacka J, Kolak M, Kiviluoto T, Arkkila P, Sirén J, Hamsten A, Fisher RM, Yki-Järvinen $\mathrm{H}$ : Genes involved in fatty acid partitioning and binding, lipolysis, monocyte/macrophage recruitment, and inflammation are overexpressed in the human fatty liver of insulin-resistant subjects. Diabetes 2007, 56:2759-2765.

23. Yen CL, Stone SJ, Koliwad S, Harris C, Farese RV Jr: Thematic review series: glycerolipids. DGAT enzymes and triacylglycerol biosynthesis. J Lipid Res 2008, 49:2283-2301.

24. Romeo S, Huang-Doran I, Baroni MG, Kotronen A: Unravelling the pathogenesis of fatty liver disease: patatin-like phospholipase domain-containing 3 protein. Curr Opin Lipidol 2010, 21:247-252.

25. Levy M, Castillo SS, Goldkorn T: nSMase2 activation and trafficking are modulated by oxidative stress to induce apoptosis. Biochem Biophys Res Commun 2006, 344:900-905.

26. Claus RA, Bunck AC, Bockmeyer CL, Brunkhorst FM, Losche W, Kinscherf R, Deigner HP: Role of increased sphingomyelinase activity in apoptosis and organ failure of patients with severe sepsis. FASEB J 2005, 19:1719-1721.

27. Samad F, Hester KD, Yang G, Hannun YA, Bielawski J: Altered adipose and plasma sphingolipid metabolism in obesity. A potential mechanism for cardiovascular and metabolic risk. Diabetes 2006 55:2579-2587

28. Håversen L, Danielsson KN, Fogelstrand L, Wiklund O: Induction of proinflammatory cytokines by long-chain saturated fatty acids in human macrophages. Atherosclerosis 2011, 202:382-393.

29. Blachnio-Zabielska AU, Pulka M, Baranowski M, Nikolajuk A, Zabielski P, Górska M, Górski J: Ceramide metabolism is affected by obesity and diabetes in human adipose tissue. J Cell Physiol 2012, 227:550-557

30. Marathe S, Kuriakose G, Williams KJ, Tabas I: Sphingomyelinase, an enzyme implicated in atherogenesis, is present in atherosclerotic lesions and binds to specific components of the subendothelial extracellular matrix. Arterioscler Thromb Vasc Biol 1999, 19:2648-2658.

31. Adiels M, Taskinen MR, Boren J: Fatty liver, insulin resistance, and dyslipidemia. Curr Diab Rep 2008, 8:60-64.

32. Kotronen A, Seppänen-Laakso T, Westerbacka J, Kiviluoto T, Arola J, Ruskeepää A-L, Yki-Jarvinen H, Orešic M: Comparison of lipid and fatty acid composition of the liver, subcutaneous and intraabdominal adipose tissue, and serum. Obesity 2010, 18:937-944.

33. Pang C, Gao Z, Yin J, Zhang J, Jia W, Ye J: Macrophage infiltration into adipose tissue may promote angiogenesis for adipose tissue remodeling in obesity. Am J Physiol Endocrinol Metab 2008, 295:E313-E322

34. Cogolludo A, Moreno L, Frazziano G, Moral-Sanaz J, Menendez C, Castañeda J, González C, Villamor E, Perez-Vizcaino F: Activation of neutral 
sphingomyelinase is involved in acute hypoxic pulmonary vasoconstriction. Cardiovasc Res 2009, 82:296-302.

35. Van BrockyIn JR, Williams JB: The control of the balance between ceramide and sphingosine-1-phosphate by sphingosine kinase: oxidative stress and the seesaw of cell survival and death. Comp Biochem Physiol B Biochem Mol Biol 2012, 163:26-36.

doi:10.1186/1476-511X-11-115

Cite this article as: Kolak et al: Expression of ceramide-metabolising enzymes in subcutaneous and intra-abdominal human adipose tissue. Lipids in Health and Disease 2012 11:115.

\section{Submit your next manuscript to BioMed Central and take full advantage of:}

- Convenient online submission

- Thorough peer review

- No space constraints or color figure charges

- Immediate publication on acceptance

- Inclusion in PubMed, CAS, Scopus and Google Scholar

- Research which is freely available for redistribution 